\title{
An Attitude Modelling Method Based on the Inherent Frequency of a Satellite Platform
}

\author{
Fan Mo ${ }^{\text {a, }}$, Xinming Tang ${ }^{\text {a }}$, Junfeng Xie ${ }^{\text {a }}$, Chao Yan ${ }^{\text {a }}$ \\ ${ }^{a}$ Research and Development Department, Satellite Surveying and Mapping Application Center, NASG, Beijing, China - (mof, \\ tangxm, xiejf, yangc)@sasmac.cn \\ Commission EuroCOW17, WG I/4
}

KEY WORDS: Satellite Attitude; Post-Process; AttModel; FFT; Inherent Frequency

\begin{abstract}
:
The accuracy of attitude determination plays a key role in the improvement of surveying and mapping accuracy for high-resolution remote-sensing satellites, and it is a bottleneck in large-scale satellite topographical mapping. As the on-board energy is constrained and the performance of an attitude-measurement device is limited, the attitude acquired is discretely sampled with a settled time interval. The larger the interval, the easier the data transmission, and the more deviation the attitude data will have. Meanwhile, several kinds of jitter frequencies have been detected in satellite platforms. This paper presents a novel attitude modelling (AttModel) method that sufficiently considers the discrete and periodic characteristics, and the attitude model built is continuous and consists of several inherent waves of different frequencies. The process of modelling includes two steps: (a) frequency detection, which uses raw gyroscope data within a period of time to detect the attitude frequencies (as the gyroscope data can actually reflect continuous, very small changes of the satellite platform), and (b) attitude modelling, which processes the attitude data that was filtered by extended Kalman filtering based on general polynomial and trigonometric polynomials, and these trigonometric polynomials are rebuilt by those frequencies detected in the first part of the modelling process. Finally, one experiment designed for verifying the effectiveness of the presented method shows that the AttModel method can reach a slightly better pointing accuracy without groundcontrol points than traditional attitude-interpolation methods.
\end{abstract}

\section{INTRODUCTION}

As more and more high-resolution remote-sensing satellites will be launched in the coming years, the accuracy of attitude determination will be a key point in expanding the application of satellites to the fields of surveying and mapping. Most remote-sensing satellites are equipped with star trackers and gyroscopes, which are recognized as providing the highest achievable accuracy (Tang, 2015). As the on-board energy is constrained and the performance of an attitude-measurement device is limited, the attitude data acquired are discretely sampled with a settled time interval. When the attitude between two adjacent discrete attitudes is required, traditionally two main types of methods can be used to obtain it (Shin, 1997). The first is the interpolation method, which uses several adjacent attitude data points around the point that needs to be calculated. The common interpolation methods include Lagrange's interpolation, cubic spline interpolation, and quaternions spherical linear interpolation (Slerp). The interpolation methods mentioned are those that are primarily used, and the Slerp could achieve the highest accuracy among them. The second method is fitting, which uses all or most of the attitude data measured within a period of time. Common fitting methods include general polynomial curve fitting and trigonometric polynomial curve fitting. A satellite equipped with low-precision attitude-measurement device will adopt this method, and general polynomial curve fitting is more commonly used than trigonometric polynomial curve fitting in the determination of satellite attitude. Compared to the abovementioned two methods, the attitude accuracy obtained may be affected by surrounding data and the time sampling interval in the interpolation method, and the accuracy cannot fit the periodic and high-frequency attitude change in the fitting method.
Considering different frequencies, attitude jitter has been detected in some remote-sensing satellites, such as the ZY-3 satellite with $0.67-\mathrm{Hz}$ jitter, ASTER with $1.6 \mathrm{~Hz}$, and Landsat-4 with $2 \mathrm{~Hz}$ (Amberg, 2013) (Tong, 2015) (Avouac, 2006) (Jiang, 2014); these different-frequency jitters may affect the attitude accuracy when the data obtained using a traditional method is between two discrete attitude values. In this paper, we present a novel attitude modelling (AttModel) method, which takes sufficient advantage of periodic changes in attitude data. The method presented includes two steps: (a) attitude analysis in the frequency domain, and (b) attitude modelling with a polynomial. Step (a) primarily obtains the inherent frequency of satellite attitude, also called the frequency window, according to the analysis of raw gyroscope data by fast Fourier transform (FFT) (John, 2006), since the gyroscope records continuous change in the satellite platform, and this continuous change could reflect obvious periodicity in the frequency domain. Step (b) models the attitude by polynomial and trigonometric fitting; the trigonometric fitting is based on frequency window. After the above two steps, we obtain a hybrid polynomial combined with several time polynomials and several trigonometric polynomials with different frequencies.

\section{ATTITUDE-MODELING METHOD}

Considering the discreteness and periodicity of attitude data, we propose applying a new method to overcome the two characteristics (Tang, 2012). To that end, this paper presents a novel attitude-modelling (AttModel) method for building a continuous attitude model based on the inherent frequency of a satellite platform.

For the reasons that the bandwidth of the signal transform channel is narrow and the performance of attitude-measurement system is limited, attitude data must be acquired at a certain 
frequency. When the frequency is low, the time interval of attitude data will be so large that it will reduce the amount of data, but that is more likely to cause low attitude precision. However, a small time interval will produce a large amount of data, and the attitude-acquirement device may not meet performance requirements that sampling in a high-frequency.

The AttModel, to some extent, could alleviate the above problems; it includes two parts: (1) analysis of attitude in the frequency domain, and (2) modelling of attitude in the time domain. In the first part, we apply the raw gyroscope data to perform the analysis. The main reason is that gyroscopes record the continuous and tiny changes in satellite platform attitude. These periodic elements of attitude will be detected by the FFT in the frequency domain. These elements can be regarded as the inherent frequencies of the satellite platform. The second part of AttModel will model the satellite attitude based on these periodic elements, which are reflected at several specific frequencies. These frequencies can be acquired for any attitude by the first part of the AttModel. [The attitude, which is processed by extended Kalman filtering (EKF) of raw gyroscopic and star sensor data (Tang, 2015), could include any amount of data; the amount is commonly more than 1000 . For example, for $\mathrm{ZY}-3$, approximately $8 \mathrm{~min}$ of data recording results in an amount of 1920.] The attitude frequencies can be constrained by these inherent frequencies. Lastly, we obtain these inherent frequencies and eliminate other frequencies, and apply the trigonometric and polynomial functions to rebuild the attitude. The entire algorithm is diagrammed in Figure 1.

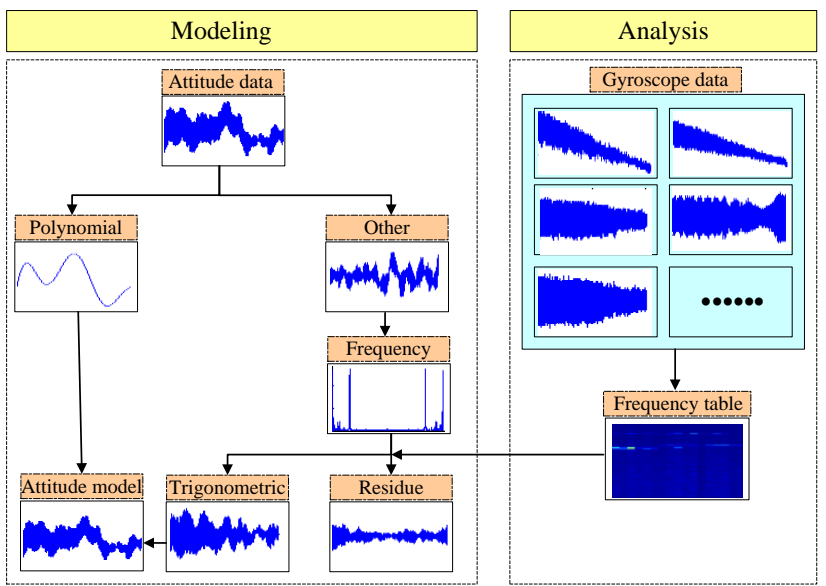

Figure 1. Attitude-modelling algorithm diagram.

\subsection{Analysis}

A Fourier transform is a mathematical tool used in signal analysis, which relates time and frequency very well. A Fourier transform expressed as a mathematical formula as follows (John, 2006):

$$
F(u)=\int_{-\infty}^{\infty} f(t) e^{-j 2 \pi u t} d t
$$

where $f(t)$ is the spatial domain value, $F(u)$ is its value in the frequency domain, $t$ is time, $u$ is frequency, and $j$ is an imaginary coefficient of a complex.

We find that a frequency spectrum reflects the proportionality of different frequencies via a Fourier transform according to the analysis of gyroscopic data. Therefore, we find the attitude frequency window $F_{\text {gyro }}(u)$ according to the frequency spectrum.

\subsection{Modelling}

As shown in Figure 1, the modelling process primarily includes four parts: polynomial fitting, frequency analysis, trigonometric fitting, and modelling. The polynomial fitting restrains the influence of a general constant value, and its mathematical formula is

$$
f(x)=a_{0}+a_{1} x+\cdots+a_{n} x^{n}
$$

where $a_{i}(i=0,1, \cdots, n)$ is the coefficient of the polynomial, $x^{i}(i=0,1, \cdots, n)$ is an independent variable, $n$ is the order of the polynomial, and $f(x)$ is a dependent variable.

After polynomial fitting, we obtain the polynomial part $f_{\text {poly }}(t)$ and other attitude part $f_{\text {other }}(t)$ should be got by original signal $f_{\text {poly }}(t)$. We apply formula (4) to process $f_{\text {other }}(t)$, and obtain a corresponding frequency $F_{\text {attitude }}(u)$ that reflects the percentage of different frequencies. The $F_{\text {model }}(u)$ can be obtained by calculation based on the frequency window $F_{g y w}(u)$, which was calculated in Equation (1) Analysis. The calculation proceeds as follows:

$$
F_{\text {model }}(u)=F_{\text {attitude }}(u) \cap F_{\text {gyro }}(u)
$$

where $F_{\text {model }}(u)$ is the frequency part after constraint, $F_{\text {attitude }}(u)$ is the corresponding frequency of $f_{\text {other }}(t), F_{g y r o}(u)$ is the frequency window, and $\cap$ is the intersection operation between them.

After obtaining $F_{\text {model }}(u)$, we use a trigonometric function to invoke the inverse Fourier transform process that will transfer the frequency data to continuous data in the spatial domain. This process mainly relies on the compound and decomposition theory of a Fourier transform (John, 2006). The process is

$$
f_{\text {cos }}(t)=c_{0}+2 \sum_{k=1}^{\infty}\left|c_{k}\right| \cos \left(2 \pi k F_{\text {model }}^{k} t+\theta_{k}\right)
$$

where $f_{c o s}(t)$ is the continuous attitude data, $c_{0}$ is a constant value, $k$ is the order of the trigonometric function (determined by $\left.F_{\text {model }}^{k}\right), c_{k}$ is the amplitude about the $k$-th order trigonometric function, $F_{\text {model }}^{k}$ is the frequency, and $\theta_{k}$ is the initial phase.

After calculating formulas (1)-(4), we complete the entire calculation process, and obtain the model by formulas (2) and (4) as follows:

$$
\begin{aligned}
\operatorname{Att}(t) & =f_{\text {poly }}(t)+f_{\text {cos }}(t) \\
& =a_{0}+a_{1} t+\cdots+a_{n} t^{n}+c_{0}+2 \sum_{k=1}^{\infty}\left|c_{k}\right| \cos \left(2 \pi k F_{\text {model }}^{k} t+\theta_{k}\right)
\end{aligned}
$$

where $A t t(t)$ is the continuous model, $t$ is time, and the other parameters are defined as in formulas (2) and (4).

The continuous model Att $(t)$ includes the linear components $f_{\text {poly }}(t)$ and the period components $f_{c o s}(t)$, and the interpolation model is continuous.

\section{EXPERIMENT AND ANALYSIS}

In order to make a comprehensive evaluation of the algorithm, we designed an experimental scheme to facilitate comparison of the AttModel to traditional methods, specifically polynomial interpolation and Slerp.

We apply the three methods to the acquisition of attitude values in a certain time period, and factor the respective attitude values into the geometric location process via a rigorous satellite imaging model. The imaging model is calibrated with both 
interior and exterior elements. By comparing the accuracy of the resulting geometric location computed by the three methods, we determine the effectiveness of the proposed method (AttModel).

\subsection{Experimental data}

We applied attitude data for the ZY-3 satellite, launched on January 9, 2012; ZY-3 is China's first civilian high-resolution stereo surveying and mapping satellite (Tang, 2015) (Tang, 2012). We randomly selected image stripe data, and the orbit ID is 00381 . There are 2188 discrete attitude values in that stripe. The time interval of adjacent discrete data is $0.25 \mathrm{~s}$. The acquisition time ranges from 97499270.07 to $97499816.82 \mathrm{~s}$. Scan area 00381 is shown in Figure 2.

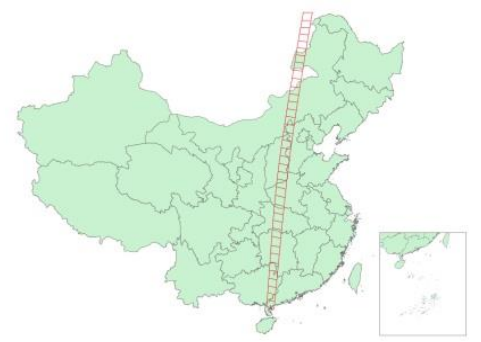

Figure 2. Scan area of experimental data.

The attitude data in the orbital coordinate system can better express satellite platform attitude changes, and the attitude data, filtered by gyroscopic and star-tracker data, are plotted in Figure 3.

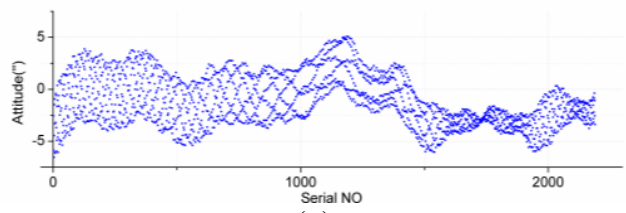

(a)

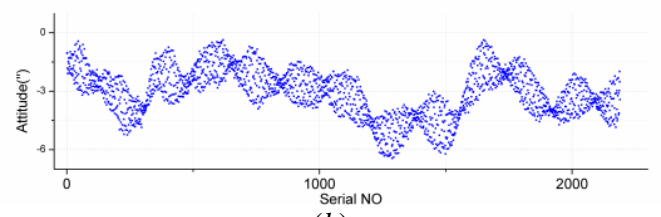

(b)

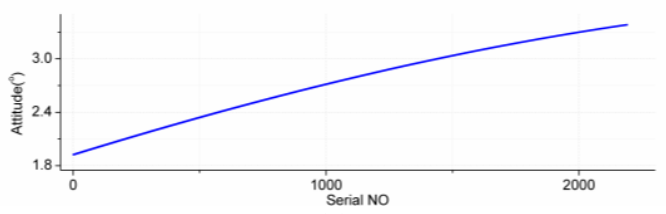

(c)

Figure 3. (a) Roll attitude data, (b) Pitch attitude data, and (c) Yaw attitude data.

As the attitude data were acquired in initial stage of satellite onboard (February, 2012), we apply the gyroscope data, which mainly include March, April and May of 2012

\subsection{Detection and analysis}

We obtained the frequency distribution spectrum of the attitude data from analysis of the three-axis, raw gyroscopic data. There are four groups of gyroscopes on the ZY-3 satellite platform, and each group includes three gyroscopes in three orthogonal axes. We apply the gyroscopic data measured by the first group of gyroscopes, GYRO-1, GYRO-3, and GYRO-5, to analyze the attitude, and obtain the frequency spectra shown in Figure 4.

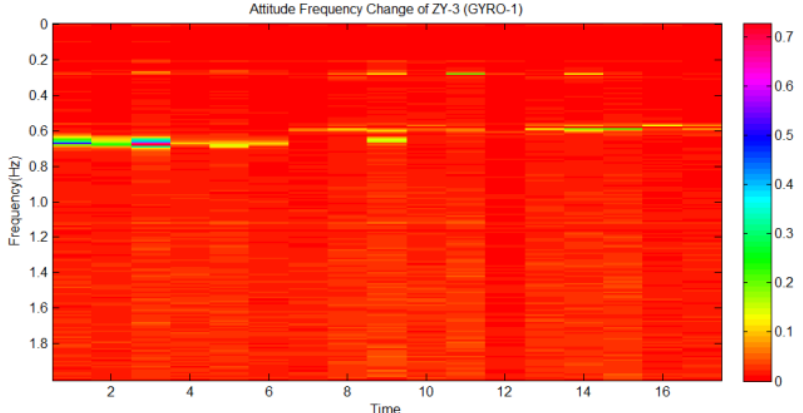

(a)

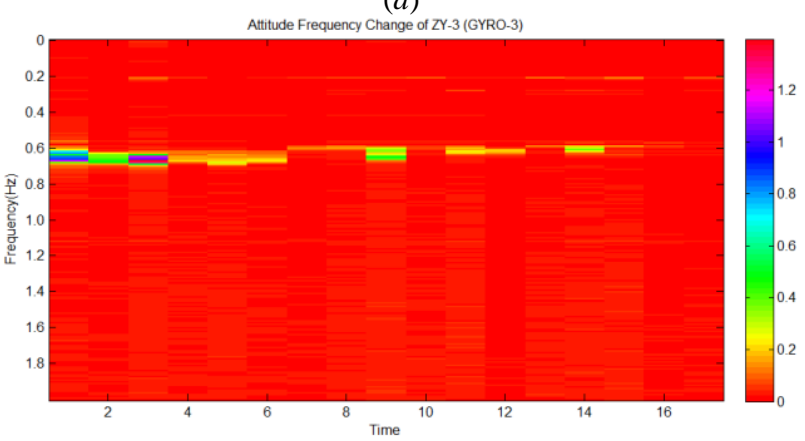

(b)

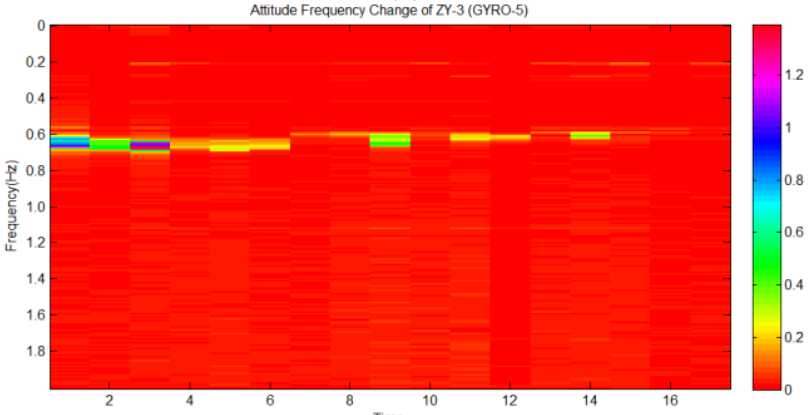

(c)

Figure 4. Attitude frequency spectra. (a) Attitude frequency spectrum of GYRO-1, (b) Attitude frequency spectrum of GYRO-3, and (c) Attitude frequency spectrum of GYRO-5

We obtain the frequency windows by analyzing the frequency spectra. The majority of satellite platform jitter is displayed in the attitude frequency spectrum. Considering that the sample frequency of the attitude-measurement system of ZY-3 is $4 \mathrm{~Hz}$, jitter measuring less than $2 \mathrm{~Hz}$ can be detected by frequency analysis (John, 2006). In addition, since low-frequencies probably cannot be detected, and have a large amplitude, their contribution to the attitude information should not be ignored. Lastly, we set the frequency windows as $0-0.3,0.56-0.7$, and $1.12-1.14 \mathrm{~Hz}$.

We obtain the frequency windows by analyzing the attitude frequency spectra, and apply them to the attitude data. Letting the frequency windows process the attitude frequencies to obtain effective values in the frequency domain, we plot the window frequencies in Figure 5.

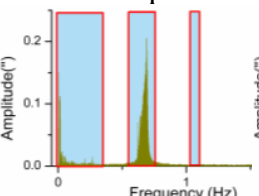

(a)

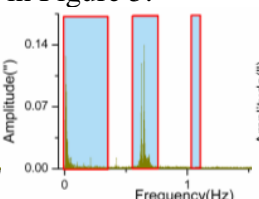

(b)

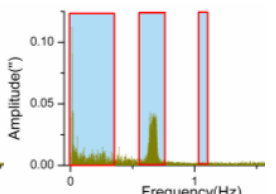

(c)
Figure 5. Window frequencies. (a) Roll, (b) Pitch, and (c) Yaw 
Figures $5(a), 5(b)$, and $5(c)$ show the three-axis frequency distribution and corresponding frequency windows for roll, pitch, and yaw, respectively. These figures show that the frequency distribution is mainly concentrated in three frequency windows: $0-0.3,0.56-0.7$, and $1.12-1.14 \mathrm{~Hz}$. These results verify that satellite platform jitter exists and the result of gyroscopic frequency analysis is true. Thus, the AttModel we have designed is feasible in theory.

\subsection{Verification and analysis}

In order to conduct an absolute evaluation of AttModel, we randomly chose ten continuous scenes of remote-sensing images in the stripe and six corresponding ground-control points (GCPs) (uniform distribution) to verify the accuracy. The images were already processed by radiation correction and their quality met production requirements. The GCPs are measured by continuously operating reference station (CORS) technology by use of GPS equipment, and their precision is better than 5 $\mathrm{cm}$.

The location results for three methods (AttModel, polynomial, and Slerp) in the longitudinal, latitudinal, and elevation directions are shown in Figure 6.

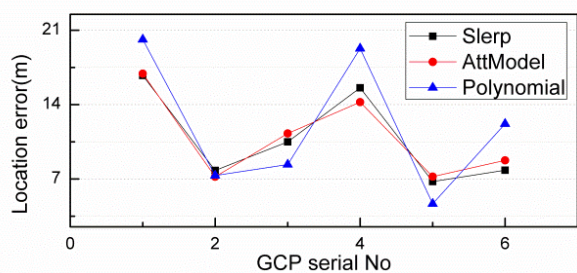

(a)

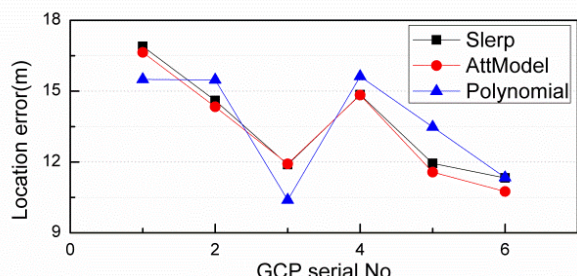

(b)

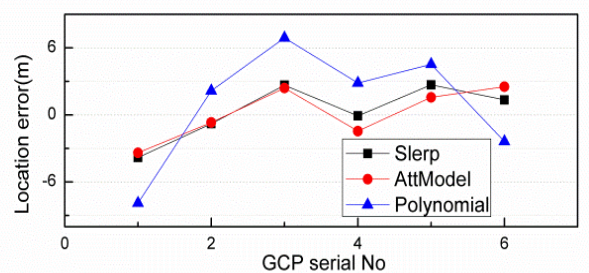

(c)

Figure 6. Location accuracy results of three methods. (a) Location results in longitudinal direction, (b) Location results in latitudinal direction, and (c) Location results in elevation direction.

The rough location accuracy can be seen in Figure 6; the quantitative location results are listed in Table 1.

Table 1. Location accuracy comparison of three methods used in this study (RMSE denotes root mean-square error).

\begin{tabular}{cccc}
\hline \hline \multirow{2}{*}{ Method } & \multicolumn{3}{c}{ RMSE (m) } \\
\cline { 2 - 4 } & $\mathrm{X}$ & $\mathrm{Y}$ & $\mathrm{Z}$ \\
\hline Polynomial & 13.359 & 13.797 & 4.98 \\
\hline \hline
\end{tabular}

\begin{tabular}{cccc}
\hline Slerp & 11.555 & 13.732 & 2.282 \\
\hline AttModel & 11.528 & 13.503 & 2.185 \\
\hline \hline
\end{tabular}

We find that the location accuracy of the AttModel method is superior to the polynomial method and slightly superior to the Slerp method based on the results of the above-described tests in different directions.

\section{CONCLUSION}

In this paper, we have presented a novel satellite attitudeinterpolation method denoted AttModel, and its location accuracy has been verified by experiment based on ZY-3 satellite attitude data. Compared to traditional methods such as polynomial fitting and Slerp, the AttModel exhibits higher precision and robustness. This paper offers an alternative attitude-interpolation method for high-resolution remotesensing satellite locations that could be optimized through the refinement of frequency windows and of the characteristics of the attitude data.

\section{ACKONWLEDGEMENTS}

The authors thank the Satellite Surveying and Mapping Application Center of China for contributing the experimental data. This paper was substantially supported by the National Natural Science Foundation of China (Project Nos. 41301525 and 41571440), and the Special Scientific Research Fund of Public Welfare Surveying and Mapping Profession of China (Project Nos. 201412007 and 201512012). The authors would like to thank the anonymous reviews for their constructive comments and suggestions.

\section{REFERENCES}

Amberg V., Dechoz C., and Lebegue L., 2013, In-flight attitude perturbances estimation: application to PLEIADES-HR satellites. Proc Spie, pp. 886612-1-886612-9.

Avouac J.P., Ayoub F., and Leprince S., et al., 2006, The 2005, M w 7.6 Kashmir earthquake: Sub-pixel correlation of ASTER images and seismic waveforms analysis. Earth Planet Science Latters, 249, pp. 514-528.

Jiang Y.H., Zhang G., and Tang X.M., et al., 2014, Detection and Correction of Relative Attitude Errors for ZY1-02C. IEEE Transactions on Geoscience and Remote Sensing, 52(12), pp. 7674-7683.

Proakis John and Manolakis Dimitris, 2006, Digital Signal Processing: Principles, Algorithms, and Applications, Fourth Edition, USA: Prentice Hall Publishers, New Jersey, pp. 165215 .

Shin D., Pollard J.K., and Muller J.P., 1997, Accurate geometric correction of ATSR images. IEEE Transaction on Geoscience Remote sensing, 35, pp. 997-1006.

Tang X.M., Zhou P., and Zhang G., et al., 2015, Verification of ZY-3 Satellite Imagery Geometric Accuracy Without Ground Control Points. IEEE Geoscience and Remote Sensing Letters, 12, pp. 2100- 2104.

Tang X.M., Xie J.F., 2012, Overview of the key technologies for high-resolution satellite mapping. International Journal of Digital Earth, 5, pp. 228-240. 
Tang X.M, Xie J.F., and X. Wang, W., et al., 2015, HighPrecision Attitude Post-Processing and Initial Verification for the ZY-3 Satellite. Remote Sensing, 7, pp. 111-134.

Tong X.H., Xu Y.S., and Ye Z., et al., 2015, Attitude Oscillation Detection of the ZY-3 Satellite by Using Multispectral Parallax Images. IEEE Transactions on Geoscience and Remote Sensing, 53, pp. 3522-3534. 